\title{
Antioxidant and antimicrobial effects of Geranium extracts as flavoring agents in some prepared foods
}

\author{
Eman Abo Zaid, Nahed Yousef, Eman Mohamed and Rabab Salem
}

Faculty of Home Economic, Al-Azhar University, Tanta, Egypt

\section{III}

Background: Essential oils and their fragrance compounds are a very important part of industry. Geranium extracts are used in numerous health disorders and medical conditions and has been validated as commercial exploitation in many industrial branches. Aim: Test the effect of geranium extract against spoilage and pathogenic bacteria, and test if it can be used for the development of novel systems for food preservation. Materials and Methods: Geranium steam extract and geranium n-Butanol extract were used as a natural flavor in food products. Results: The selected Geranium extracts were used as natural sanitizers to reduce and control pathogen contamination or the growth of native microflora. The inclusion of geranium extracts altered the antioxidant properties of the ice cream and jelly candy samples. Due to some limitations of the extract impacting on texture and color of the product, the extract was recommended to use at the lowest level. Conclusion: Geranium was found to be a good source of alternative for the future flavors of ice cream and jelly candy.

Keywords: Key words: Antioxidant; Antimicrobial; Hydro distillation; Pelargonium graveolens; Supercritical $\mathrm{CO}_{2}$

Editor-in-Chief: Prof. M.L. Salem, PhD - Article DOI: 10.21608/JCBR.2021.56898.1111 